\title{
The function and evolution of the restriction factor viperin in primates was not driven by lentiviruses
}

\author{
Efrem S Lim ${ }^{1,2,5}$, Lily I Wu' ${ }^{2}$ Harmit S Malik 3,4 and Michael Emerman ${ }^{2,3^{*}}$
}

\begin{abstract}
Background: Viperin, also known as RSAD2, is an interferon-inducible protein that potently restricts a broad range of different viruses such as influenza, hepatitis $C$ virus, human cytomegalovirus and West Nile virus. Viperin is thought to affect virus budding by modification of the lipid environment within the cell. Since HIV-1 and other retroviruses depend on lipid domains of the host cell for budding and infectivity, we investigated the possibility that Viperin also restricts human immunodeficiency virus and other retroviruses.

Results: Like other host restriction factors that have a broad antiviral range, we find that viperin has also been evolving under positive selection in primates. The pattern of positive selection is indicative of Viperin's escape from multiple viral antagonists over the course of primate evolution. Furthermore, we find that Viperin is interferoninduced in HIV primary target cells. We show that exogenous expression of Viperin restricts the LAI strain of HIV-1 at the stage of virus release from the cell. Nonetheless, the effect of Viperin restriction is highly strain-specific and does not affect most HIV-1 strains or other retroviruses tested. Moreover, knockdown of endogenous Viperin in a lymphocytic cell line did not significantly affect the spreading infection of HIV-1.
\end{abstract}

Conclusion: Despite positive selection having acted on Viperin throughout primate evolution, our findings indicate that Viperin is not a major restriction factor against HIV-1 and other retroviruses. Therefore, other viral families are likely responsible for the evolutionary signatures of positive selection in viperin among primates.

\section{Background}

Antiviral proteins engaged in virus-host interactions are often locked in evolutionary "arms-races", which have been referred to as "Red Queen" conflicts. Viral infections continuously exert immense selective pressures on the host antiviral proteins to evolve adaptively. The signatures of these evolutionary conflicts can be inferred by observing signals of adaptive evolution (also called positive selection) in antiviral genes that result from repeated episodes of Darwinian selection due to past viral infections [1]. Often, the exact amino acids under positive selection can describe the sites and domains involved in host-virus interaction [2-4]. Thus, a detailed look at the evolutionary trajectory of an antiviral gene

\footnotetext{
*Correspondence: memerman@fhcrc.org

${ }^{2}$ Division of Human Biology, Fred Hutchinson Cancer Research Center, 1100 Fairview Ave. N, Seattle, WA 98109-1024, USA

${ }^{3}$ Division of Basic Sciences, Fred Hutchinson Cancer Research Center, Seattle, WA, USA

Full list of author information is available at the end of the article
}

can provide valuable information about the viral pressures that shaped host evolution.

Viperin (Virus inhibitory protein, endoplasmic reticulum-associated, interferon-inducible, also known as RSAD2) is a host protein with broad antiviral activity (reviewed in [5-7]). Viperin inhibits the release of a wide range of viruses in cell culture including Influenza A virus [8], Hepatitis C virus [9-11], and Japanese Encephalitis virus [12]. Moreover, viperin knockout mice demonstrate the importance of this protein in controlling West Nile Virus pathogenesis in vivo [13]. In the case of human cytomegalovirus (HCMV), Viperin has been reported not only to inhibit the expression of late viral gene products [14] but also to enhance HCMV infectivity by remodeling the cellular actin cytoskeleton [15].

The precise mechanism of the broad-spectrum antiviral function of Viperin remains unclear. However, one model for Viperin antiviral activity links lipid raft disruption to the restriction of Influenza virus release [16].

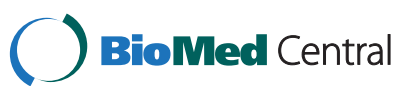


Lipid rafts are sphingolipid- and cholesterol-enriched microdomains on the plasma membrane that have also been implicated in a number of processes including membrane signaling, polarization, and immunological synapse function $[17,18]$. Additionally, lipid rafts also play an important role in the entry and assembly stages of viral replication $[17,19]$. Moreover, the host sterol biosynthesis pathway is downregulated in response to viral infections as part of the innate immune response via type I interferon signaling [20]. Viperin has also been shown to directly inhibit farnesyl diphosphate synthethase (FPPS), a cellular enzyme critically involved in the biosynthesis of isoprenoid-derived lipids [16]. This suggests that the disruption of cellular lipid raft formation may represent a generalized host defense against viruses. As lipid rafts are thought to be sites of assembly and budding for HIV and other retroviruses [17,21-23], we investigated whether Viperin restricts HIV-1 and other retroviruses.

We find that viperin, like other host restriction factors against viruses, has evolved under positive selection in primates. We find that Viperin inhibits the release of the LAI strain of HIV-1. However, we show that HIV-1 and SIV strains have intrinsic differences in their sensitivity to Viperin, and most are unaffected by over-expression of Viperin. Furthermore, we did not see an effect of Viperin knockdown on HIV-1 growth. Collectively, our findings suggest that Viperin is not a major restriction factor against HIV-1 and retroviruses, and thus its positive selection must have been driven by other viral pathogens.

\section{Results}

\section{Viperin has been evolving under positive selection} in primates

A recurring theme of host restriction factors is that they exhibit a strong signature of positive selection [24]. Given the remarkable breadth of viruses restricted by Viperin $[5,6,14,16]$, we hypothesized that viperin might also be evolving under positive selection. To investigate this possibility, we sequenced the viperin gene from 18 species of primates and obtained 2 sequences of prosimian viperin from Genbank (Figure 1A and Additional file 1: Figure S1). Together, these primate species span around 60 million years of divergence. The phylogeny constructed from the primate viperin sequences was congruent with the generally accepted primate phylogeny [25] confirming that the sequences are orthologous. There was no evidence of recombination as ascertained by a GARD analysis [26].

In order to determine the lineage-specific pressures on the primate viperin gene, we performed a free-ratio analysis using the PAML program suite [27], which allows an independent assignment of omega $(\mathrm{dN} / \mathrm{dS})$ ratios to each evolutionary branch of the primate phylogeny, where $d N / d S$ ratios $>1$ are indicative of positive selection. Several branches of the phylogeny within the New World monkeys, Old World monkeys, and hominoids showed dN/dS ratios $>1$ (Figure 1A, bold branches). For instance, the branches leading up to Spider Monkey and FLM have $\mathrm{dN} / \mathrm{dS}$ ratios $>1$, indicative of positive selection. To test whether Viperin was subject to episodic or constant selective pressures over primate evolution, we compared the likelihood ratios of the free-ratio model (Figure 1B, Model 1) where all branches were allowed to have their own independent $\mathrm{dN} / \mathrm{dS}$, versus a model where the entire phylogeny had the same $\mathrm{dN} / \mathrm{dS}$ value (Figure $1 \mathrm{~B}$, Model 0 ). We found that the free-ratio model fit the data better although this was marginally significant $(\mathrm{p}=0.08)$. We therefore conclude that primate viperin has been under ancient, episodic positive selection.

We also performed a maximum likelihood analysis using codeml from the PAML program suite [27] that allows for different $\mathrm{dN} / \mathrm{dS}$ ratios across individual codons, and found strong evidence that the viperin gene has been evolving under positive selection in primates (Figure 1C). In order to determine which domain (s) in Viperin are responsible for the signal of positive selection, we examined each domain separately (the $\mathrm{N}$-terminal alpha helix domain, a short middle region, the Radical S-adenosylmethionine (SAM) domain and a flexible C-terminal domain (Figure 1D)). While the $\mathrm{N}$-terminal alpha helix was not under positive selection, the middle region, Radical SAM domain and C-terminal flexible domain showed signs of positive selection with high confidence (Figure 1D). In particular, five amino acid positions exhibit strong signals of positive selection (corresponding to residues 42, 51, 142,145, 352 in human Viperin). These five amino acid residues were independently confirmed to be under positive selection with strong significance by randomeffect likelihood (REL) analyses (data not shown) [28]. Importantly, removal of these five amino acids leads to loss of the signature of positive selection from the analyses (Figure 1C), validating that the majority of the positive selection was acting on these sites. The dispersed nature of these positively selected residues is reminiscent of other broadly acting antiviral genes like Protein Kinase R (PKR), wherein escape from viral antagonism drives the positive selection of PKR [29]. This is in contrast to other restriction factors like TRIM5alpha, where a cluster of positive selectively selected residues identifies the viral specificity domains [4]. Therefore, we conclude that viperin has evolved under positive selection, likely to escape viral antagonism by a variety of viral lineages over the course of primate evolution. 


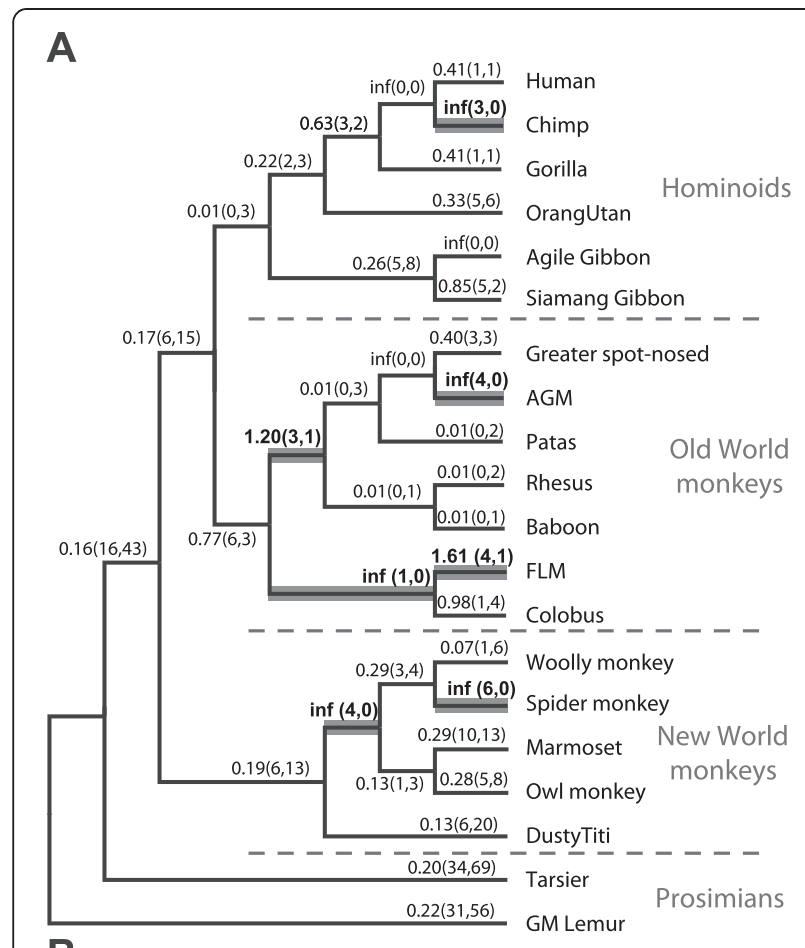

B

Likelihood ratio test statistics for models of variable selective pressure along branches

\begin{tabular}{|c|c|c|c|c|c|}
\hline \multicolumn{2}{|r|}{ Viperin } & InI & $2 \ln \lambda$ & df & $p$-value \\
\hline \multicolumn{2}{|c|}{$\begin{array}{l}\text { Model } 0 \\
\text { (Same dN/dS ratio for all branches) }\end{array}$} & -3602.55 & & & \\
\hline \multicolumn{2}{|c|}{$\begin{array}{l}\text { Model } 1 \\
\text { (Different dN/dS ratio for each branch) }\end{array}$} & -3577.75 & 49.61 & 37 & $<0.081$ \\
\hline \multicolumn{6}{|c|}{146149} \\
\hline & $\begin{array}{r}4753 \\
09\end{array}$ & $\overbrace{}^{146}$ & & & $9^{352}$ \\
\hline Viperin & $\begin{array}{l}\text { Alpha } \\
\text { Helix }\end{array}$ & Radical SAM & & min & \\
\hline
\end{tabular}

Likelihood ratio test statistics for models of variable selective pressure among sites of Viperin

\begin{tabular}{|c|c|c|c|}
\hline Viperin & $2 \ln \lambda$ & df & $p$-value \\
\hline Full gene (aa 1-361) & 26.65 & 2 & $<0.001$ \\
\hline Full gene $(\Delta 47,53,146,149,352)$ & 0.56 & 2 & $>0.755$ (NS) \\
\hline \multicolumn{4}{|l|}{ D } \\
\hline Viperin region analyzed & $2 \ln \lambda$ & df & $p$-value \\
\hline Alpha helix (aa 1-43) & 0 & 2 & 1 (NS) \\
\hline Middle domain (aa 44-77) & 16.34 & 2 & $<0.003$ \\
\hline Radical SAM domain (aa 78-262) & 7.22 & 2 & $<0.028$ \\
\hline$C$ terminal (aa 263-361) & 12.07 & 2 & $<0.003$ \\
\hline
\end{tabular}

\section{Viperin inhibits HIV-1 Lai virus release}

Given the broad antiviral range of Viperin, we wished to investigate whether Viperin might also be relevant to restricting HIV-1 infection. We first studied whether Viperin is expressed at the protein level in HIV-1 target
Figure 1 Positive selection of primate viperin. (A) Cladogram of 20 primate viperin genes sequenced for the evolutionary analyses. Free ratio analysis in PAML was used to calculate the $\omega(\mathrm{dN} / \mathrm{dS})$ ratios of individual branches. The corresponding $\omega$ ratios are shown above each branch, and the number of non-synonymous changes and synonymous changes are indicated in parentheses. Branches with $\omega>1$ are highlighted in bold. In the case of no observed synonymous changes, the $\omega$ ratio could not be calculated (indicated by 'inf'). (B) Likelihood ratio test statistics for models of variable selective pressures along branches of primate viperin genes are shown, using a comparison between $\mathrm{MO}$ (same dN/dS ratio for all branches) and M1 (different dN/dS ratio for each branch, free-ratio). (C) A schematic of Viperin protein domain structure is shown. Residues under positive selection with high confidence $(P>0.95)$ are indicated in symbols above the protein. The table summarizes the likelihood ratio test statistics for models of variable selective pressure among viperin sites (M7 vs M8). Similar results were obtained in a comparison of M1 (neutral) versus M2 (selection) (data not shown). The amino acid positions are annotated in reference to the human Viperin sequence. (D) The table summarizes likelihood ratio test statistics performed between the M7 (neutral) and M8 (selection) models for the individual protein domains of viperin gene from 20 primate species.

cells. Primary CD4+ T cells and monocytes were isolated from peripheral blood mononuclear cells of two donors and treated with interferon $\beta$ for twenty hours. We found that both primary CD4+ T cells and monocytes express endogenous Viperin after induction with interferon (Figure 2A), but expression levels were undetectable in the absence of interferon.

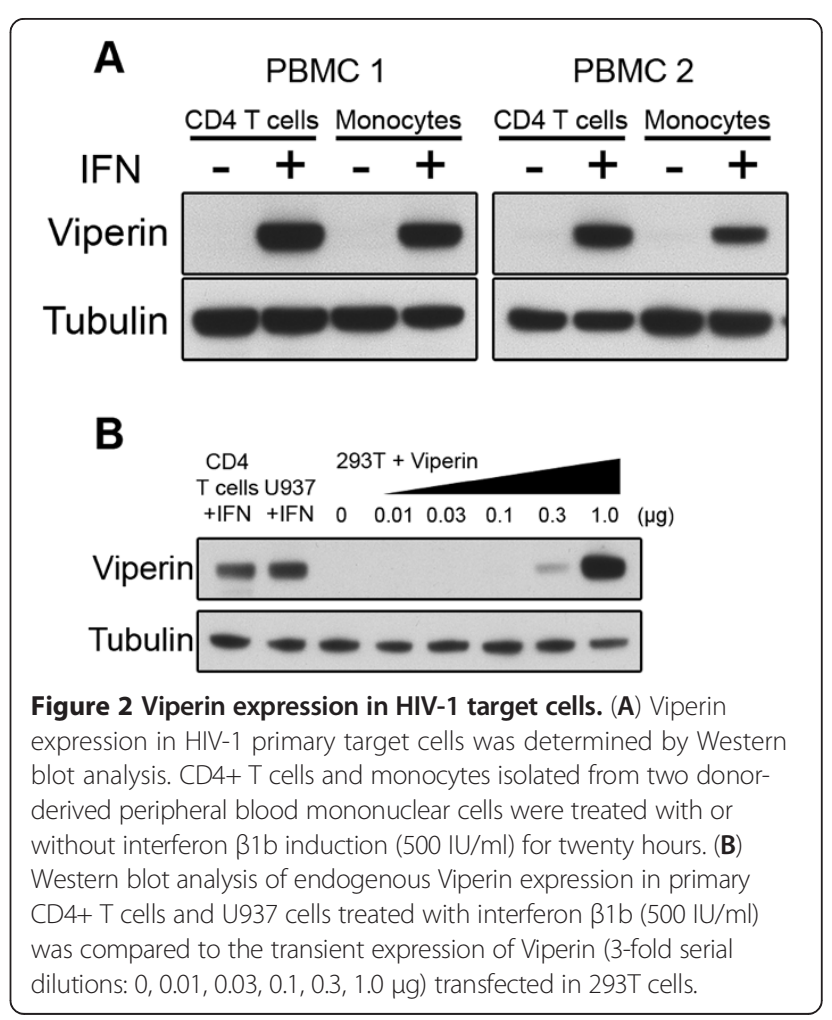




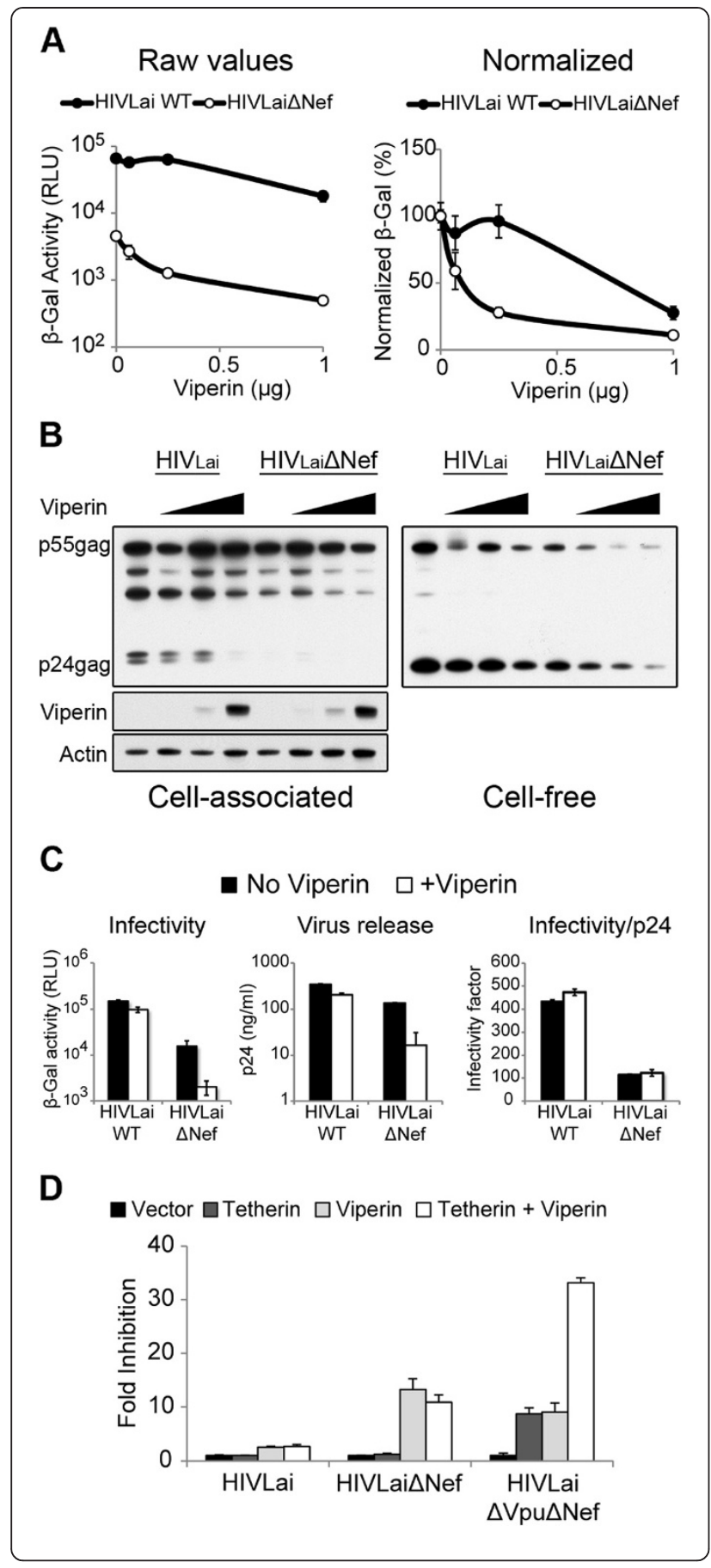

Given that Viperin is under positive selection and expressed in HIV-1 primary target cells after interferon induction, we investigated whether Viperin restricts HIV-1. To begin these studies, we first compared levels of endogenous Viperin expression with levels achieved by transfection of the cloned human viperin gene into $293 \mathrm{~T}$ cells. We found that untransfected 293T cells express undetectable levels of endogenous Viperin. However, the transient expression of Viperin in 293T cells transfected
Figure 3 Effect of Viperin on virus release. (A) The effect of viperin was measured by the infectious virus yield. 293 T cells cotransfected with $200 \mathrm{ng}$ of HIVLai or HIVLai $\Delta$ Nef with serial dilutions of human viperin was titered by infecting TZM.BL indicator cells. The infectivity readout by $\beta$-galactosidase activity measured in relative light units (RLU) is shown on the left, and the respective viruses were normalized to $\beta$-galactosidase activity in the absence of viperin as shown on the right. Error bars indicate standard deviations from three infection replicates; this data are representative of five independent experiments. (B) Western blot analysis was performed on the cell-free virus and cellular extracts, and probed with a-p24 antibody. Viperin expression in the cellular extracts is shown, and actin was probed as a loading control. This blot is representative of four independent experiments. (C) The effect of Viperin on the specific infectivity of virus particles was calculated. HIVLai or HIVLai $\Delta$ Nef virus from 293T cells co-transfected with or without Viperin (700 ng) was titered by infecting TZM.BL indicator cells as shown on the left. Virus release in the cell-free supernatant was quantified by p24 ELISA as shown in the middle. The specific infectivity was calculated as ratio of $\beta$-galactosidase activity (RLU) over the amount of p24 (ng/ml), as shown on the right. Error bars indicate standard deviations of triplicate infections; the data are representative of at least three independent experiments. (D) Virus yield from 293T cells co-transfected with a combination of Tetherin (50 ng) or Viperin (700 ng) was titered on TZM.BL cells. Fold inhibition was calculated in comparison to virus yield in absence of Tetherin/Viperin. Error bars indicate standard deviations of triplicate infections; the data are representative of three independent experiments.

with between 0.3 and $1 \mu \mathrm{g}$ of DNA bracketed the amount of endogenous Viperin expression in primary $\mathrm{CD} 4+\mathrm{T}$ cells and U937 cells when induced with interferon (Figure 2B). Therefore, in subsequent studies, we used amounts of the plasmid encoding the human viperin gene that gave levels of Viperin expression just below and just above the levels expressed in primary cells.

We tested whether exogenous Viperin expression could restrict HIV-1 by co-transfecting 293T cells with a fulllength HIV-1 Lai provirus with increasing amounts of the human viperin gene. Additionally, we tested HIV-1 Lai lacking a nef gene, since Nef has been implicated in modulating cellular cholesterol levels [30,31]. We measured the antiviral activity of Viperin by infecting TZM.BL indicator cells with released virus, and assaying for $\beta$ galactosidase reporter activity (See Methods). We found that wild-type HIV-1 virus was marginally affected at low amounts of Viperin, but was inhibited at the highest dose of Viperin (Figure 3A, closed circles). Consistent with the known defect on virion infectivity in the absence of $\mathrm{Nef}$ [32], the HIV $\Delta$ Nef virus had a lower infectivity even in the absence of Viperin as measured by the $\beta$-galactosidase activity (Figure 3A left, compare closed circles and open circles at $0 \mu \mathrm{g}$ viperin). Despite that initial observation, the HIV $\Delta$ Nef virus was restricted further by viperin in a dosedependent manner (Figure 3A, open circles). To compare the degree of restriction between the two viruses, we normalized the $\beta$-galactosidase reporter activity of each virus 
to their measurements in the absence of Viperin (Figure $3 \mathrm{~A}$ right). We observed that the wildtype HIV virus was only restricted at the highest levels of Viperin expression, whereas the HIV $\Delta$ Nef virus was more sensitive to Viperin restriction than wildtype HIV, even at the lower levels of Viperin expression.

Because Viperin restricts influenza virus at the step of virus release [16] and HCMV by inhibiting the production of viral structural proteins [14], we investigated whether HIV-1 production and/or release is affected by Viperin by Western blotting for cell-associated and cellfree Gag proteins. We hypothesized that if Viperin affects HIV production; we expected to see a decrease in intracellular p55gag expression that correlates with a decrease in cell-free p24gag. Conversely, if Viperin affects virus release, we would see lower levels of cell-free p24gag while levels of p55gag would remain unchanged.

We found that cell-associated HIV-1 p55gag for both WT and $\Delta$ Nef virus was only marginally affected by the expression of Viperin (Figure 3B). Moreover, cell-free levels of p24gag from wild type HIV-1 were modestly affected by the expression of Viperin (Figure 3B) in a manner consistent with a slight decrease in the amount of supernatant HIV p24gag when measured with an enzyme linked immunosorbent assay (ELISA) assay (Figure 3C, middle). However, Viperin expression showed a drastic reduction in cell-free HIV $\Delta$ Nef p24gag (Figure 3B, right), with only a small effect on intracellular p55gag levels (Figure 3B, left). This suggests that Viperin affects release of HIV $\Delta$ Nef virus.

Since Viperin might also be affecting the quality of the virus particles, we quantified the specific infectivity of virus particles by measuring the ratio of infectious titer to relative particle production (by p24 ELISA). Consistent with other studies, we found that wildtype $\mathrm{HIV}$ virus was more infectious than HIV $\Delta$ Nef virus (Figure 3C). However, viperin expression did not affect the specific infectivity (infectivity divided by p24gag) of either wildtype HIV virus or HIV $\Delta$ Nef virus particles (Figure $3 \mathrm{C}$, right), indicating that the Viperin-mediated restriction of HIV-1 is not due to a reduction in viral infectivity.

Since Viperin seemed to affect virus release, we compared Viperin restriction to that of Tetherin, a wellcharacterized host restriction factor that inhibits virus release [33,34]. Virus restriction by a combination of Viperin and Tetherin expression was roughly additive (Figure 3D). Furthermore, the response of Viperin and Tetherin is different since HIV-1 Vpu abrogates Tetherin restriction but has no effect on Viperin restriction, whereas HIV-1 Nef abrogates Viperin restriction (Figure 3D). These results suggest that Viperin restricts HIV-1 release by a mechanism that is distinct from the pathway used by Tetherin.

\section{Most HIV strains, SIVs and retroviruses are resistant to Viperin restriction}

To examine the breadth of Viperin restriction on HIV-1 we tested several strains of HIV-1 for their susceptibility to Viperin restriction on virus release. The proviruses were deleted of their nef gene to exclude the possible confounding effects of Nef specificity. Consistent with the earlier experiments (Figure $3 \mathrm{C}$ ), HIVLai $\Delta$ Nef virus release was inhibited by human Viperin. However, virus release of the HIV $\Delta$ Nef NL4-3, SF162 (both HIV-1 subtype B) and Q23-17 strains (HIV-1 subtype A) were unaffected by Viperin expression (Figure 4A). To verify these observations, we performed a Western blot analysis comparing the cell-associated and cell-free HIV-1 Gag protein levels. In contrast to the dose-dependent inhibition of HIVLai $\Delta$ Nef virus release, cell-free HIVNL4-3 $\Delta$ Nef virus release remained unaffected (Figure 4B). While there was an observable effect on intracellular HIVNL4-3 $\Delta$ Nef virus p55 levels, this difference was not reflected in the cell-free Gag p24 levels or the ELISA assay. In addition, we tested an HIV-1 vector encoded from a codon-optimized Gag-pol sequence called pCNC-SynGP [35]. We observed that the cellfree HIV-1 pCNC-SynGP Gag was unaffected by Viperin expression. Instead, cell-associated p55gag protein production was slightly increased in the presence of Viperin expression. As for the HIV $\Delta$ Nef SF162 and Q23-17 strains, there were no significant effects on cellfree p24gag or cell-associated p55gag expression levels. One exception is a noticeable decrease in the partially processed, cell-associated, HIV $\Delta$ Nef SF162 p40gag levels. However, while Viperin might have a subtle effect on the intracellular Gag levels of certain HIV $\Delta$ Nef strains, the difference was not reflected in the cell-free virus or measured in the ELISA assay. Thus, it appears that Viperin does not significantly impact the virus release of most HIV $\Delta$ Nef strains tested except for the HIV-1 Lai strain.

We next investigated the ability of Viperin to restrict related simian immunodeficiency virus (SIV). In addition to their nef gene deletion, the proviruses were also pseudotyped with VSV-G so that the entry of all viruses would be equal. Using an infectivity assay, we found that SIVmac239 $\Delta$ Nef was as sensitive to Viperin as HIV$1 \mathrm{Lai} \Delta \mathrm{Nef}$ (Figure $4 \mathrm{C}$, open squares). However, SIVagmTAN1 $\Delta$ Nef, SIVcpzTAN3.1 $\Delta$ Nef and HIV-2ROD9 $\Delta$ Nef were resistant to Viperin restriction. Since Viperin did not appear to restrict the majority of primate lentiviruses we tested, we also examined two additional divergent retroviruses - murine leukemia virus (MLV) and feline immunodeficiency virus (FIV). In contrast to the control HIV $\triangle$ Nef Lai virus, MLV and FIV were unaffected by Viperin expression (Figure 4D), indicating that Viperin does not generally restrict retroviruses. Thus, while 


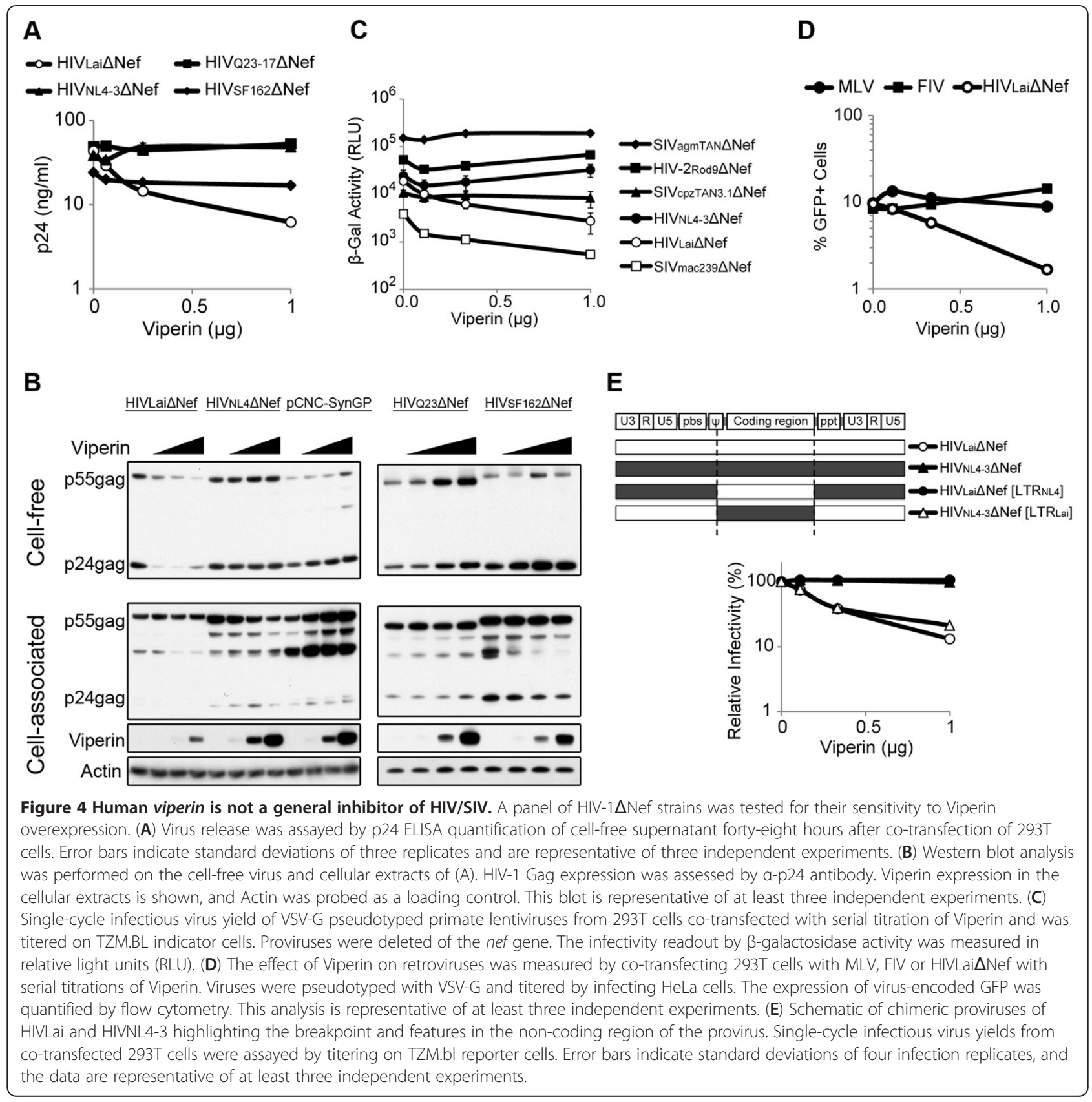

Viperin may inhibit a limited subset of primate lentivirus strains (HIV-1Lai and SIVmac239, for example), the majority of HIV-1 strains, SIVs and retroviruses that we tested are not affected by Viperin expression.

The difference in restriction profiles between Lai and NL4-3 strains was unexpected since NL4-3 is a recombinant virus of NY5 and Lai strains [36]. We attempted to map the viral determinant of Viperin sensitivity by constructing a series of chimeric proviruses between Lai and NL4-3 strains and tested them in an infectivity assay. While we expected that changes in Gag proteins previously associated with virus release might be involved, chimeric proviruses within Gag failed to identify a determinant within Gag (data not shown). Instead, we found that even when all coding regions were swapped between the Lai and NL4-3 strains of HIV-1, the sensitivity to Viperin restriction still mapped to Lai sequences outside of the coding region (Figure 4E). That is, when the coding region of HIVLai was expressed in the context of HIV-1 NL4-3 non-coding region, sensitivity to Viperin restriction was lost. However, when we inserted the non-coding region of HIV-1 Lai including the LTRs, 5' packaging region, and the PPT into HIV-1 NL4-3, then the virus was sensitive to inhibition by 
Viperin (Figure 4E). Thus, human Viperin displays differential restriction specificities against related HIV-1, which is dictated entirely by non-coding regions of the provirus. We conclude, therefore, that the restriction by exogenous Viperin of HIV-1 is likely due to threshold effects of expression, rather than due to direct interactions of Viperin with viral components.

\section{Knockdown of endogenous Viperin in a cell line does not affect HIV-1 Lai}

A more critical test of the physiological role of Viperin on HIV-1 replication is to change levels of endogenous Viperin in cells where spreading infections can be performed. To determine the effect of endogenous Viperin on HIV-1, we sought to knock-down Viperin expression with shRNAs. Unfortunately, we were unable to obtain suitable stable or transient knockdown in primary $\mathrm{T}$ cells (data not shown). Many $\mathrm{T}$ cell lines such as SupT1 cells also do not express Viperin after interferon induction (data not shown). However, CD4+ U937 monocytic cells do express Viperin after interferon induction (Figure 2B). Using a stably transduced shRNA construct, we were able to partially knockdown expression of Viperin in U937 cells (Figure 5A). U937 cells that were either knocked-down for Viperin (shRNAVip) or transduced with a control shRNA (shCON) were infected with HIV-1LAI and HIV-1LAI $\Delta$ Nef at a multiplicity of infection of 0.5 . Infections were done in the presence of interferon to induce Viperin expression. Viral supernatant was collected periodically over 11 days, and spreading infection was monitored by p24 ELISA. We found that there was no significant difference between WT and $\Delta$ Nef virus growth in the cells knocked-down

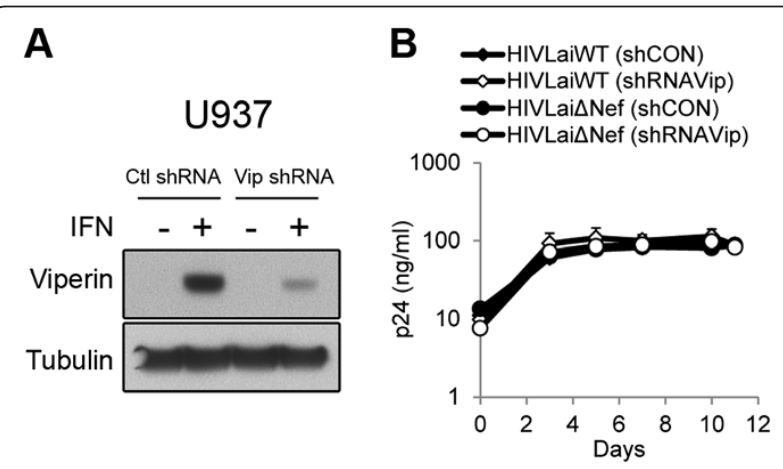

Figure 5 Analyses of endogenous Viperin activity. (A) Viperin expression in U937 cells stably transduced with empty pGIPZ shRNA vector (Ctl) or Viperin targeting shRNA (Vip) was analyzed by Western blot twenty hours after interferon $\beta 1 \mathrm{~b}$ treatment (500 IU/ml). (B) Spreading infection of wildtype HIVLai or HIVLai $\Delta$ Nef was quantified by p24 ELISA at indicated time points after infecting shRNA-transduced U937 cells at an moi of 0.5. Cells were maintained in interferon $\beta 1 \mathrm{~b}(500 \mathrm{lU} / \mathrm{ml})$ for the duration of the experiment. The data are representative of at least three independent experiments. for Viperin expression (Figure 5B). Considering that the HIVLai $\Delta$ Nef is the most sensitive strain in Viperin overexpression experiments (Figuresa 2 and 4), these results suggest that endogenous levels of Viperin do not affect spreading infection by HIV-1.

\section{No functional divergence in lentiviral restriction among primate Viperin orthologs}

Most of the experiments that we have carried out were using the human viperin allele. However, since viperin evolves rapidly under positive selection, we might not be accurately capturing the potential ability of Viperin proteins to restrict lentiviruses. The species-specificity of action is one of the key features that have emerged from the study of rapidly evolving restriction factors. To address the possibility that the human Viperin might not accurately capture the restrictive potential of Viperin, we carried out two experiments to measure any functional divergence between primate Viperin orthologs that may have arisen from the positive selection.

First, we tested 5 additional Viperin orthologs against the HIVLai $\Delta$ Nef (Figure 6A). We found that all six Viperin orthologs are able to restrict this virus to approximately the same extent, despite some variation in the degree of restriction. This means that the positive selection of viperin does not manifest a functional difference in the degree of restriction of HIVLai $\Delta$ Nef. Second, we compared the human and rhesus orthologs against a panel of viruses to assess whether we could discern any key restriction differences between these two Viperin orthologs (Figure 6B). It is notable that human versus rhesus differences have been found in many positively selected restriction factors that have been tested so far $[4,29,37]$. However, in the case of Viperin, we found no significant differences between the restriction profiles of human and rhesus Viperin.

These results imply that Viperin's lack of restriction of the majority of lentiviruses and retroviruses tested is general to primate viperin genes. Moreover, this finding strongly implies that gain or loss of lentivirus restriction is not correlated with the dramatic evolutionary changes we observed in the viperin gene in primates.

\section{Discussion}

Primate Viperin is not a lentiviral restriction factor

The restriction factor Viperin recognizes and restricts a wide diversity of viruses, including both single-stranded RNA and double-stranded DNA viruses [5-7]. This broad repertoire of antiviral activity prompted us to investigate Viperin's restrictive activity against retroviruses, specifically the primate lentivirus lineage. We found that Viperin is highly interferon-induced in primary target cells of HIV. Viperin overexpression is able to inhibit HIV-1 Lai replication by affecting virus release. 
A
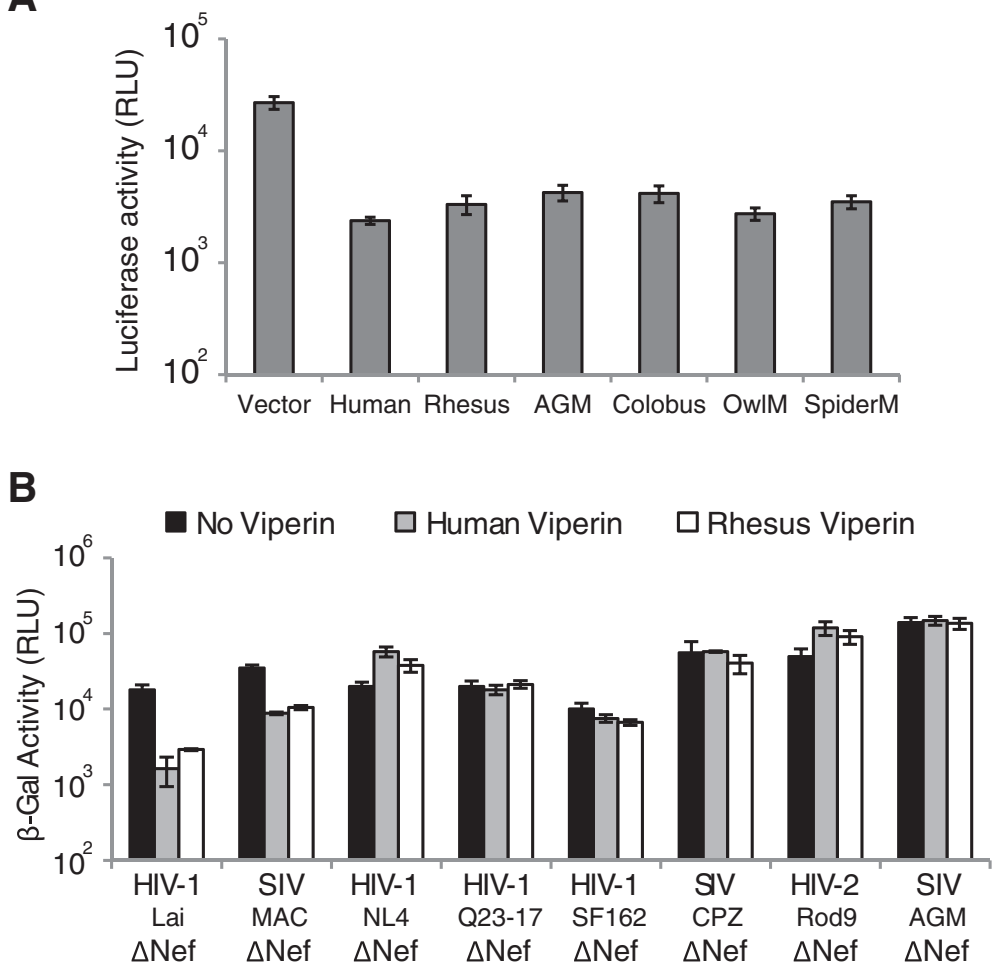

Figure 6 The antiviral activity of primate Viperin orthologs was assayed. (A) 293T cells were co-transfected with HIVLai $\Delta$ Nef that encodes a luciferase reporter gene (200 ng) and the indicated primate Viperin (700 ng). Virus yield was measured by infecting SupT1 cells and assayed for luciferase expression. Error bars represent standard deviations of four infection replicates and are representative of three independent experiments. (B) Single-cycle infectious virus yield of VSV-G pseudotyped primate lentiviruses from 293T cells co-transfected with $700 \mathrm{ng}$ of human Viperin, rhesus Viperin or empty vector was titered on TZM.BL indicator cells. The infectivity readout by $\beta$-galactosidase activity was measured in relative light units (RLU). Error bars indicate standard deviations of four infection replicates, and are representative of at least three independent experiments.

However, most other strains of HIV-1, SIV and other retroviruses are unaffected by primate Viperin orthologs. Finally, we could not find an effect on spreading infections of HIV-1 Lai in U937 cells after knockdown of endogenous Viperin levels. Therefore, we conclude that Viperin is not a major restriction factor against HIV-1 and other primate lentiviruses. These results emphasize the fact that broadly acting innate host defense genes do, nonetheless, have viral specificity that goes beyond their escape from viral antagonism.

Lipid rafts play an important role in virus replication and are actively regulated as part of the host response to viral infection [20]. As Viperin inhibits Influenza virus release by impairing the lipid metabolic pathway enzyme FPPS resulting in the disruption of lipid rafts [16], we expected that it would have a broad antiviral role in inhibited viruses that bud through lipid rafts, in a way similar to how Tetherin affects many different enveloped viruses that bud through the plasma membrane [38]. However, importantly, we did not find this to be the case since HIV was generally resistant to the effects of Viperin. Thus, these data argue that it is overly simplistic to characterize all viral lipid raft interactions as equivalent, but rather there are likely important differences in the lipid requirements for budding of different virus families. It may be important that unlike HIV, influenza assembly at lipid rafts does not involve the ESCRT machinery [39].

A previous study that showed that poly I:C-induced Viperin had a subtle effect on HIV-1 infection in astrocytes [40]. However, our findings are not consistent with an effect of Viperin on HIV-1 replication in general. Although other retroviral restriction factors appear to be active in all cells tested, it is possible that the antiviral effects of Viperin are cell-specific and would be active in primary cells that were not able to be tested in this study. Furthermore, it is formally possible that all of the retroviruses tested encode an antagonist of Viperin that abrogates its action. However, virus-host antagonism should show species-specificity [1]; we believe that this is very unlikely because viperin cloned from a wide range of different primates showed equivalent activities against HIV-1 and diverse retroviruses (Figure 6 and data not shown), and Viperin expression is not affected 
by co-transfection with proviruses (data not shown). Nonetheless, the fact that we did find two lentiviral proviruses encoded by HIV- 1 Lai and SIVmac239 that were inhibited by transfection of viperin, suggests that the pathway used by Viperin must at least peripherally intersect with lentiviral production.

Our findings of Viperin restriction of the Lai strain of HIV-1 but not the NL4-3 strains are unexpected since NL4-3 is a recombinant virus of NY5 and Lai strains [36]. We have mapped the genetic basis of the susceptibility difference to a non-coding region of the virus. Moreover, we found that HIV-1Lai with a deletion in Nef was more sensitive to Viperin than a full-length provirus. Complementation of the Lai strain of HIV-1 $\Delta \mathrm{Nef}$ provirus with Nef in trans partially restored the resistance to Viperin (data not shown). One possible explanation is that LTR promoter efficiency and the presence of Nef may affect viral Gag production in a manner that renders it sensitive to Viperin. Alternatively, we believe it is more likely that less infectious viral combinations are more sensitive to perturbations caused by exogenous expression of Viperin. Nonetheless, considering that most strains of HIV-1, SIVs (excluding SIVmac239), tested are resistant to Viperin, we favor the more parsimonious conclusion that Viperin is not a significant player in the immune defense against lentiviruses. Moreover, the results describe here serve as an important caution that over-expression systems with single isolates cannot be relied on to functionally identify and characterize restriction factors.

\section{Insight into viperin function from its positive selection}

Antagonistic genetic conflicts between hosts and viruses have driven rapid adaptive evolution of antiviral protein which is characteristic of many retroviral restriction factors [24] as well as other antiviral factors that target a broad range of viruses $[29,41]$. Like many host restriction factors, we find that viperin has been evolving under positive selection in primates. The signatures of rapid evolution in viperin may provide valuable information about the mechanism by which it restricts this broad repertoire of viruses and likely avoids viral antagonism. This is analogous to the dynamics of the host restriction factor Tetherin, where the highest recurrent signal of positive selection corresponds to the amino acid that is a determinant for antagonism by Nef [3]. In the antagonist-driven scenario, we speculate that the amino acid residues under positive selection on Viperin might have been driven by pressures to evade viral antagonists and would be indicative of sites directly involved in viral protein interactions. In this regard, the evolution of primate Viperin may provide valuable clues to virus families that have driven the positive selection of Viperin throughout primate evolution [1,41] by finding which viruses encode antagonists to Viperin with a specificity that is specified by the amino acids in Viperin that are under positive selection. A promising candidate would be Japanese encephalitis virus which encodes an unidentified viral antagonist that degrades Viperin in a proteasome-dependent mechanism [12].

\section{Conclusion}

We document an ancient, episodic and recurrent history of adaptive evolution in Viperin over primate evolution. However, despite the fact that Viperin restricts a wide range of other virus families [15], it does not have a major effect on HIV-1 and other lentiviruses, and therefore, the positive selection in viperin was likely driven by selective pressures imposed by virus families other than the lentiviruses.

\section{Methods}

\section{Plasmids}

Human Viperin was cloned from human cDNA derived from 293T cells, and inserted into a retroviral expression vector $\mathrm{pLPCX}$ as an untagged construct. The five primate Viperin orthologs were similarly cloned from cDNA into the pLPCX retroviral expression vector as untagged constructs. HIVLai, HIVLai $\Delta$ Nef and HIVLai $\Delta$ Vpu $\Delta$ Nef, SIVagmTAN $\Delta$ Env $\Delta$ Nef were described previously $[3,42]$. HIVNL4-3 $\Delta$ Nef was obtained from the NIH AIDS Research and Reference Reagent Program, 11100. HIVSF62 $\Delta$ Nef was generated by fill-on of the XhoI site (nt 8576) resulting in a $2 \mathrm{bp}$ frameshift mutation in the Nef open reading frame of the full length HIV-1SF162 provirus [43]. HIV-1Q23-17 $\Delta$ Nef was constructed by introducing a luciferase gene in place of $\mathrm{Nef}$ into the full length HIV-1 Q23-17 provirus [44]. SIVcpz $\Delta$ Nef was generated by introducing a luciferase gene in place of Nef into the full length SIVcpzTAN3.1 provirus [45] (NIH AIDS Research and Reference Reagent Program, 11100) by overlapping PCR between the NdeI and NheI region and sequence verified. HIV$2 \operatorname{Rod} 9 \Delta$ Env $\Delta$ Nef was a gift from Masahiro Yamashita, and SIVmac239 $\Delta$ Env $\Delta$ Nef was a gift from David Evans [46]. pGIPZ vector-based control shRNA or shRNA targeting Viperin mRNA (hairpin construct: TGCTG TTGACAGTGAGCGCGATGAAAGACTCCTACCTTA TTAGTGAAGCCACAGATGTAATAAGGTAGGAGTC TTTCATCTTGCCTACTGCCTCGGA) were purchased from FHCRC RNAi core facility.

\section{Viral infectivity assay}

$293 \mathrm{~T}$ cells were seeded at $1.67 \times 10^{5}$ cells $/ \mathrm{ml}$ in 12 -well plates, and DNA was transfected with TransIT LT-1 (Mirius) according to the manufacturer's recommendations. The total amount of DNA in all transfections was maintained constant with appropriate empty vectors. 
Forty-eight hours after transfection, supernatant was collected, filtered through a $0.2 \mu \mathrm{M}$ filter and serially diluted for the following infectivity assay. SupT1 cells at $2.5 \mathrm{x}$ $10^{5}$ cells $/ \mathrm{ml}$ in 96-well plates were as described previously [3], or TZM.bl cells at $1.0 \times 10^{5}$ cells $/ \mathrm{ml}$ in 96 -well plate were as described previously [42]. The $\beta$ Galactosidase activity was detected using the GalactoStar system (Applied Biosystems) according to the manufacturer's recommendations.

\section{Virus release p24 ELISA}

Virus were serially diluted and measured by HIV-1 p24 antigen capture assay (Advanced BioScience Lab Inc) and detected with QuantaRed enhanced chemifluorescent HRP substrate (Thermo Scientific) according to the manufacturer's protocol.

\section{Western blotting}

Western blot analysis was performed as described previously $[3,42]$ with the following antibodies: HA-specific antibody (Babco), anti-Viperin (Enzo Life Sciences), antiactin (Sigma-Aldrich), anti-tubulin (Sigma-Aldrich), and HIV-1 p24 antibody (NIH Aids Research and Reference Reagent Program, 183-H12-5C) [47]. Primary antibodies were detected with a corresponding horseradish peroxidaseconjugated secondary antibody.

\section{PBMC isolation and separation}

Patient pall filters were obtained from Puget Sound Blood Center. PBMCs were isolated by standard ficoll histopaque gradient methods. Monocytes and CD4+ T cells were isolated by Human CD14 selection and CD4+ magnetic bead isolation (EasySep), and the isolation purity (>97-99\%) was confirmed by flow cytometry staining (BD Pharmingen). Monocytes were maintained in RPMI containing 10\% FBS. CD4+ T cells were activated with $2.5 \mu \mathrm{g} / \mathrm{ml} \mathrm{PHA}$ and $20 \mathrm{U} / \mathrm{ml}$ IL-2 for 3 days before interferon treatment. Monocytes and CD4+ T cells were treated with $500 \mathrm{IU} / \mathrm{ml}$ human interferon $\beta 1 \mathrm{~b}$ for 20 hours, followed by western blot analysis on total cell lysates.

\section{Spreading infectivity assay}

U937 cells stably transduced with either a Viperintargeting shRNA or control shRNA constructs were infected with a wild type HIV-1Lai virus or HIV-1Lai $\Delta$ Nef at a moi of 0.5. Cells were washed with PBS three times and maintained in media containing $500 \mathrm{IU} / \mathrm{ml}$ human interferon $\beta 1 \mathrm{~b}$ throughout the course of the experiment. Supernatant was collected at indicated time points and virus was quantified by p24 ELISA.

\section{Sequencing of primate viperin genes}

The viperin genes from the following primates were amplified from RNA isolated from cell lines obtained from Coriell Cell Repositories (Camden, NJ): chimpanzee (Pan troglodytes), gorilla (Gorilla gorilla), Sumatran orangutan (Pongo pygmaeus), Siamang gibbon (Hylobates syndactylus), agile gibbon (Hylobates agilis), rhesus macaque (Macaca mulatta), greater white-nosed monkey (Cercopithecus nictitans), kikuyu colobus (Colobus guereza kikuyuensis), Francois' leaf monkey (FLM) (Trachypithecus francoisi), spider monkey (Ateles geoffroyi), owl monkey (Aotus trivirgatus), dusty titi monkey (Callicebus moloch) and woolly monkey (Lagothrix lagotricha). Human (Homo sapiens), African green monkey (Chlorocebus aethiops) and Baboon (Papio anubis) Viperin were amplified by reverse transcription-PCR (RT-PCR) from an RNA extract of 293 T cells, COS-7 cells and B-LCL cells respectively. Viperin was amplified by RT-PCR with a OneStep RT-PCR kit (Qiagen), and the cDNA derived was directly sequenced. Viperin was amplified with "forward" primer (5'-ATGTGGGGTGCTTACACCTGCTG CTTTTGCTG-3') or (5'-ATGTGGGTACTCACGCCTG CTGCTTTTGCTG-3') in combination with "reverse" primer (5'-CTACCAATCCAGCTTCAGATCAGCCTTA CTC-3') or (5'-CTACCAATCCAGCTTCAGATCAGCC TTACTC-3'). Sequences for prosimian grey mouse lemur (Microcebus murinus) and tarsier (Tarsius syrichta) viperin gene were obtained by tblastx search on the NCBI database from cont1.216710 (ABDC01216711.1) and contig1.93320 (ABRT010093321.1) respectively.

\section{Sequence analysis}

DNA sequences were aligned by ClustalX [48] and were edited manually. The amino acid positions are annotated in reference to the human Viperin sequence. A phylogeny of viperin genes was constructed from DNA sequences with ClustalX by the neighbor-joining method using the Jukes Cantor method of correction and with PhyML [49] by the maximum-likelihood method. The two methods yielded trees with identical topologies. Maximum-likelihood analysis was performed with CODEML from the PAML suite of programs [27] as previously described [2,3]. Sequence alignments were obtained when the data were fitted with an F61 model of codon frequency, and consistent results were obtained when the data were fitted with an F3 $x 4$ model of codon frequency. Viperin sequences were fitted to NSsites models that disallowed (NSsites model 1 and 7) or permitted (NSsites model 2 and 8) positive selection. Likelihood ratio tests were performed to evaluate whether permitting codons to evolve under positive selection gave a better fit to the data. A cutoff of posterior probability of $\mathrm{p}>0.95$ was implemented in these analyses (M8) to identify amino acid residues having evolved under positive selection. Analyses were also validated with REL from the HyPhy package [50]. Free ratio analysis in PAML was used to calculate the $\omega(\mathrm{dN} / \mathrm{dS})$ ratios 
of individual branches. Likelihood ratio test statistics was performed for models of variable selective pressures along branches of primate viperin genes between M0 (same $\mathrm{dN} / \mathrm{dS}$ ratio for all branches) and M1 (different $\mathrm{dN} / \mathrm{dS}$ ratio for each branch). The degree of freedom is equal to one less than the total number of branches in the phylogeny.

\section{Nucleotide sequence accession numbers}

The sequences of the 18 primate viperin genes have been entered into the GenBank database under accession numbers NM_080657, JQ437821 to JQ437837.

\section{Additional file}

\section{Additional file 1: Figure 1. Amino acid alignment of 20 primate} viperin genes sequenced for the evolutionary analyses. The protein domain structure is shown above the alignment, and residues under positive selection are highlighted in bold. The amino acid positions are annotated in reference to the human Viperin sequence.

\section{Competing interests}

The authors declare that they have no competing interests.

\section{Acknowledgments}

The following reagents were obtained through the NIH AIDS Research and Reference Reagent Program, Division of AIDS, NIAID, NIH: HIV-1 p24 Monoclonal Antibody (183-H12-5C) from Dr. Bruce Chesebro and Kathy Wehrly, HIV-1SF 162 (276) from Dr. Jay Levy, HIV-1NL4-3 (11100) from Drs. Haili Zhang, Yan Zhou, and Robert Siliciano. We thank Julie Overbaugh for the HIV-1 Q23-17 proviral construct, Masahiro Yamashita for HIV-2Rod9 $\Delta$ Env $\Delta$ Nef, Ned Landau for SIVagmTAN $\Delta$ Env $\Delta$ Nef, and David Evans for the SIVmac239 Env $\Delta$ Nef construct; the FHCRC Genomics and RNAi Shared Resources; and Alex Compton and Lucie Etienne for comments on the manuscript. This work was supported by NIH grant R01 Al30937 (to M.E.) and an NSF Career grant (to H.S.M.). H.S.M. is an Early-Career Scientist of the Howard Hughes Medical Institute. E.S.L. is supported by the University of Washington Helen Riaboff Whiteley Graduate Fellowship.

\section{Author details}

'Department of Microbiology, Fred Hutchinson Cancer Research Center, University of Washington, Seattle, WA, USA. ${ }^{2}$ Division of Human Biology, Fred Hutchinson Cancer Research Center, 1100 Fairview Ave. N, Seattle, WA 98109-1024, USA. ${ }^{3}$ Division of Basic Sciences, Fred Hutchinson Cancer Research Center, Seattle, WA, USA. ${ }^{4}$ Howard Hughes Medical Institute, Fred Hutchinson Cancer Research Center, Seattle, WA, USA. ${ }^{5}$ Present address: Department of Molecular Microbiology and Pathology \& Immunology, Washington University School of Medicine, St. Louis, MO 63110, USA.

\section{Authors' contributions}

ESL performed all experiments and except for Figure 5 which was done by LIW. ESL, HSM and ME designed experiments, analyzed data, and wrote the manuscript. All authors read and approved the final manuscript.

Received: 28 March 2012 Accepted: 26 June 2012

Published: 26 June 2012

\section{References}

1. Emerman M, Malik H: Paleovirology-modern consequences of ancient viruses. PLOS Biol 2010, 8:e1000301.

2. Lim ES, Fregoso Ol, McCoy CO, Matsen FA, Malik HS, Emerman M: The Ability of Primate Lentiviruses to Degrade the Monocyte Restriction Factor SAMHD1 Preceded the Birth of the Viral Accessory Protein Vpx. Cell Host Microbe 2012, 11:194-204.
3. Lim ES, Malik HS, Emerman M: Ancient adaptive evolution of tetherin shaped the functions of Vpu and Nef in human immunodeficiency virus and primate lentiviruses. J Virol 2010, 84:7124-7134.

4. Sawyer SL, Wu LI, Emerman M, Malik HS: Positive selection of primate TRIM5alpha identifies a critical species-specific retroviral restriction domain. Proc Natl Acad Sci U S A 2005, 102:2832-2837.

5. Mattijssen S, Pruijn GJ: Viperin, a key player in the antiviral response. Microbes Infect 2012, 14(5):419-426.

6. Seo JY, Yaneva R, Cresswell P: Viperin: A Multifunctional, InterferonInducible Protein that Regulates Virus Replication. Cell Host Microbe 2011, 10:534-539.

7. Fitzgerald KA: The interferon inducible gene: Viperin. J Interferon Cytokine Res 2011, 31:131-135.

8. Wang $S$, Wu X, Pan T, Song W, Wang Y, Zhang F, Yuan Z: Viperin inhibits Hepatitis C Virus replication by interfering with the binding of NS5A to host protein hVAP-33. J Gen Virol 2012, 93:83-92.

9. Helbig KJ, Eyre NS, Yip E, Narayana S, Li K, Fiches G, McCartney EM, Jangra RK, Lemon SM, Beard MR: The antiviral protein viperin inhibits hepatitis $\mathrm{C}$ virus replication via interaction with nonstructural protein $5 \mathrm{~A}$. Hepatology 2011, 54:1506-1517.

10. Helbig KJ, Lau DT, Semendric L, Harley HA, Beard MR: Analysis of ISG expression in chronic hepatitis $\mathrm{C}$ identifies viperin as a potential antiviral effector. Hepatology 2005, 42:702-710.

11. Jiang D, Guo H, Xu C, Chang J, Gu B, Wang L, Block TM, Guo JT: Identification of three interferon-inducible cellular enzymes that inhibit the replication of hepatitis C virus. J Virol 2008, 82:1665-1678.

12. Chan $Y L$, Chang $T H$, Liao $C L$, Lin $Y L$ : The cellular antiviral protein viperin is attenuated by proteasome-mediated protein degradation in Japanese encephalitis virus-infected cells. J Virol 2008, 82:10455-10464.

13. Szretter K, Brien JD, Thackray LB, Virgin HW, Cresswell P, Diamond MS: The interferon-inducible gene viperin restricts West Nile virus pathogenesis. J Virol 2011, 85(22):11557-11566.

14. Chin KC, Cresswell P: Viperin (cig5), an IFN-inducible antiviral protein directly induced by human cytomegalovirus. Proc Natl Acad Sci U S A 2001, 98:15125-15130.

15. Seo JY, Yaneva R, Hinson ER, Cresswell P: Human Cytomegalovirus Directly Induces the Antiviral Protein Viperin to Enhance Infectivity. Science 2011, 32:1093-1097.

16. Wang $X$, Hinson ER, Cresswell P: The interferon-inducible protein viperin inhibits influenza virus release by perturbing lipid rafts. Cell Host Microbe 2007, 2:96-105.

17. Ono A, Freed EO: Role of lipid rafts in virus replication. Adv Virus Res 2005, 64:311-358.

18. Simons $K$, Gerl MJ: Revitalizing membrane rafts: new tools and insights. Nat Rev Mol Cell Biol 2010, 11:688-699.

19. Mañes S, del Real G, Martínez-A C: Pathogens: raft hijackers. Nat Rev Immunol 2003, 3:557-568.

20. Blanc M, Hsieh WY, Robertson KA, Watterson S, Shui G, Lacaze P Khondoker M, Dickinson P, Sing G, Rodriguez-Martin S, et al: Host defense against viral infection involves interferon mediated down-regulation of sterol biosynthesis. PLoS Biol 2011, 9:e1000598.

21. Nitta T, Kuznetsov $Y$, McPherson A, Fan H: Murine leukemia virus glycosylated Gag (gPr80gag) facilitates interferon-sensitive virus release through lipid rafts. Proc Natl Acad Sci U S A 2010, 107:1190-1195.

22. Pickl WF, Pimentel-Muiños FX, Seed B: Lipid rafts and pseudotyping. J Virol 2001, 75:7175-7183.

23. Ono A, Freed EO: Plasma membrane rafts play a critical role in HIV-1 assembly and release. Proc Natl Acad Sci U S A 2001, 98:13925-13930

24. Meyerson NR, Sawyer SL: Two-stepping through time: mammals and viruses. Trends Microbiol 2011, 19:286-294.

25. Perelman $\mathrm{P}$, Johnson WE, Roos C, Seuanez HN, Horvath JE, Moreira MA, Kessing B, Pontius J, Roelke M, Rumpler Y, et al: A molecular phylogeny of living primates. PLoS Genet 2011, 7:e1001342.

26. Kosakovsky Pond SL, Posada D, Gravenor MB, Woelk CH, Frost SD: GARD: a genetic algorithm for recombination detection. Bioinformatics 2006, 22:3096-3098

27. Yang Z: PAML: a program package for phylogenetic analysis by maximum likelihood. Comput Appl Biosci 1997, 13:555-556.

28. Kosakovsky Pond SL, Frost SD: Not so different after all: a comparison of methods for detecting amino acid sites under selection. Mol Biol Evol 2005, 22:1208-1222. 
29. Elde NC, Child SJ, Geballe AP, Malik HS: Protein kinase $R$ reveals an evolutionary model for defeating viral mimicry. Nature 2009, 457:485-489.

30. Wout AB Van 't, Swain JV, Schindler M, Rao U, Pathmajeyan MS, Mullins Jl, Kirchhoff F: Nef induces multiple genes involved in cholesterol synthesis and uptake in human immunodeficiency virus type 1-infected T cells. J Virol 2005, 79:10053-10058.

31. Zheng YH, Plemenitas A, Fielding CJ, Peterlin BM: Nef increases the synthesis of and transports cholesterol to lipid rafts and HIV-1 progeny virions. Proc Natl Acad Sci U S A 2003, 100:8460-8465.

32. Arhel NJ, Kirchhoff F: Implications of Nef: host cell interactions in viral persistence and progression to AIDS. Curr Top Microbiol Immunol 2009, 339:147-175.

33. Neil SJ, Zang T, Bieniasz PD: Tetherin inhibits retrovirus release and is antagonized by HIV-1 Vpu. Nature 2008, 451:425-430.

34. Van Damme N, Goff D, Katsura C, Jorgenson RL, Mitchell R, Johnson MC, Stephens EB, Guatelli J: The interferon-induced protein BST-2 restricts HIV-1 release and is downregulated from the cell surface by the viral Vpu protein. Cell Host Microbe 2008, 3:245-252.

35. Ikeda Y, Takeuchi Y, Martin F, Cosset FL, Mitrophanous K, Collins M: Continuous high-titer HIV-1 vector production. Nat Biotechnol 2003, 21:569-572.

36. Adachi A, Gendelman HE, Koenig S, Folks T, Willey R, Rabson A, Martin MA Production of acquired immunodeficiency syndrome-associated retrovirus in human and nonhuman cells transfected with an infectious molecular clone. J Virol 1986, 59:284-291.

37. Evans DT, Serra-Moreno R, Singh RK, Guatelli JC: BST-2/tetherin: a new component of the innate immune response to enveloped viruses. Trends Microbiol 2010, 18:388-396.

38. Martin-Serrano J, Neil SJ: Host factors involved in retroviral budding and release. Nat Rev Microbiol 2011, 9:519-531.

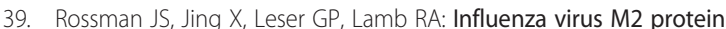
mediates ESCRT-independent membrane scission. Cell 2010, 142:902-913.

40. Rivieccio MA, Suh HS, Zhao Y, Zhao ML, Chin KC, Lee SC, Brosnan CF: TLR3 ligation activates an antiviral response in human fetal astrocytes: a role for viperin/cig5. J Immunol 2006, 177:4735-4741.

41. Patel MR, Loo YM, Horner SM, Gale M Jr, Malik HS: Convergent evolution of escape from hepaciviral antagonism in primates. PLOS Biol 2012, 10:e1001282.

42. Lim ES, Emerman M: Simian immunodeficiency virus SIVagm from African green monkeys does not antagonize endogenous levels of African green monkey tetherin/BST-2. J Virol 2009, 83:11673-11681.

43. Cheng-Mayer C, Levy JA: Distinct biological and serological properties of human immunodeficiency viruses from the brain. Ann Neurol 1988, 23(Suppl):S58-61.

44. Poss M, Overbaugh J: Variants from the diverse virus population identified at seroconversion of a clade A human immunodeficiency virus type 1-infected woman have distinct biological properties. J Virol 1999, 73:5255-5264.

45. Takehisa J, Kraus MH, Decker JM, Li Y, Keele BF, Bibollet-Ruche F, Zammit KP, Weng Z, Santiago ML, Kamenya S, et al: Generation of infectious molecular clones of simian immunodeficiency virus from fecal consensus sequences of wild chimpanzees. J Virol 2007, 81:7463-7475.

46. Regier DA, Desrosiers RC: The complete nucleotide sequence of a pathogenic molecular clone of simian immunodeficiency virus. AIDS Res Hum Retroviruses 1990, 6:1221-1231.

47. Chesebro B, Wehrly K, Nishio J, Perryman S: Macrophage-tropic human immunodeficiency virus isolates from different patients exhibit unusual V3 envelope sequence homogeneity in comparison with T-cell-tropic isolates: definition of critical amino acids involved in cell tropism. J Virol 1992, 66:6547-6554.

48. Thompson JD, Gibson TJ, Plewniak F, Jeanmougin F, Higgins DG: The CLUSTAL_X windows interface: flexible strategies for multiple sequence alignment aided by quality analysis tools. Nucleic Acids Res 1997, 25:4876-4882

49. Guindon S, Gascuel O: A simple, fast, and accurate algorithm to estimate large phylogenies by maximum likelihood. Syst Biol 2003, 52:696-704.

50. Pond SL, Frost SD, Muse SV: HyPhy: hypothesis testing using phylogenies. Bioinformatics 2005, 21:676-679. doi:10.1186/1742-4690-9-55

Cite this article as: Lim et al:: The function and evolution of the restriction factor viperin in primates was not driven by lentiviruses. Retrovirology 2012 9:55

\section{Submit your next manuscript to BioMed Central and take full advantage of:}

- Convenient online submission

- Thorough peer review

- No space constraints or color figure charges

- Immediate publication on acceptance

- Inclusion in PubMed, CAS, Scopus and Google Scholar

- Research which is freely available for redistribution 